\title{
Short-Term Steady-State Pattern Electroretinography Changes Using a Multi-Pressure Dial in Ocular Hypertensive, Glaucoma Suspect, and Mild Open- Angle Glaucoma Patients: A Randomized, Controlled, Prospective, Pilot Study
}

\author{
Jeremy J. Kudrna (D) - Tanner J. Ferguson · Russell J. Swan • \\ Steven J. Ferguson - Brandon J. Baartman • Adam R. Bleeker • \\ Ramu G. Sudhagoni · John P. Berdahl · Michael D. Greenwood \\ Received: August 13, 2020 / Accepted: September 4, 2020 / Published online: September 16, 2020 \\ (C) The Author(s) 2020
}

\section{ABSTRACT}

Introduction: This study evaluates the effects of the multi-pressure dial (MPD) on steady-state pattern electroretinography (ss-pERG) parameters. The study is a randomized, controlled,

Digital Features To view digital features for this article go to https://doi.org/10.6084/m9.figshare.12911957.

J. J. Kudrna $(\bowtie)$

Department of Ophthalmology, Penn State College

of Medicine, Hershey, PA, USA

e-mail: jkudrna@pennstatehealth.psu.edu

T. J. Ferguson

Cole Eye Institute, Cleveland, OH, USA

R. J. Swan

Vance Thompson Vision, Bozeman, MT, USA

S. J. Ferguson

Dunes Eye Consultants, Dakota Dunes, SD, USA

B. J. Baartman

Vance Thompson Vision, Omaha, NE, USA

A. R. Bleeker

University of South Dakota Sanford School of

Medicine, Sioux Falls, SD, USA

R. G. Sudhagoni

HemoSonics, LLC, Charlottesville, VA, USA

J. P. Berdahl

Vance Thompson Vision, Sioux Falls, SD, USA

M. D. Greenwood

Vance Thompson Vision, West Fargo, ND, USA prospective, pilot trial in a private practice setting with ocular hypertensive (OHT), glaucoma suspect, and open-angle glaucoma (OAG) subjects.

Methods: This study included nine patients (64 \pm 9.0 years, nine female) with OHT, glaucoma suspect, or mild OAG. One eye of each subject was randomized to receive negative periocular pressure, while the contralateral eye served as the intrasubject control through the goggle without negative pressure. The Diopsys High Contrast Sensitivity ss-pERG protocol was conducted on both eyes of each subject while wearing the MPD device. Application of negative periocular pressure was set at $50 \%$ of baseline intraocular pressure for each study eye.

Results: Following $2 \mathrm{~h}$ of negative periocular pressure application, the difference in MagnitudeD (MagD) from baseline for eyes randomized to receive negative periocular pressure $(+0.17$ versus -0.26$)$ was statistically significant $(p=0.023)$. Over the same period, the change in MagD/Magnitude (MagD/Mag ratio) from baseline for eyes randomized to receive negative periocular pressure was also higher ( +0.14 versus -0.16$)$, compared to the control eyes, approached significance $(p=0.059)$.

Conclusions: Following $2 \mathrm{~h}$ of MPD wear, the measured MagD and MagD/Mag ratio improved compared to control, suggesting that negative periocular pressure application to the anterior globe can lead to short-term improvement in one measure of retinal ganglion cell function. 
Keywords: Glaucoma management; Multipressure dial; Steady-state pattern electroretinogram

\section{Key Summary Points}

Glaucomatous diseases are a leading cause of blindness worldwide and characterized by early atrophy of retinal ganglion cells (RGC); while intraocular pressure (IOP) reduction is currently the only clinically validated therapy for glaucoma management, early detection and novel approaches to glaucoma diagnosis and management remain atop the challenges facing clinicians today.

This study utilized a steady-state pattern electroretinography (ss-pERG), a clinical tool used to monitor early RGC dysfunction, and a multi-pressure dial (MPD), which consists of a pair of pressure-sensing goggles that separately encloses the periorbital areas of each eye with tubing that individually connects to a programmable pump that allows for IOP reduction in a controlled and predictable manner.

Included in this study were nine patients with the same documented diagnosis in both eyes of either ocular hypertension, glaucoma suspect, or mild open-angle glaucoma; one eye of each subject was randomized to receive negative periocular pressure from the MPD for $2 \mathrm{~h}$, while the contralateral eye served as the intrasubject control through the goggle without negative pressure.

We hypothesized that the ss-pERG values indicating RGC functionality, namely MagD and MagD/Mag ratio, would improve in the study eye and not in the fellow eye following $2 \mathrm{~h}$ of negative periocular pressure application from an MPD.
The results supported our hypothesis suggesting that short-term negative periocular pressure application from an MPD, and the corresponding titrated reduction in IOP, can lead to acute improvement in one measure of RGC function.

\section{DIGITAL FEATURES}

This article is published with digital features to facilitate understanding of the article. To view digital features for this article go to https://doi. org/10.6084/m9.figshare.12911957.

\section{INTRODUCTION}

Glaucomatous diseases are a leading cause of blindness worldwide $[1,2]$. Although glaucoma can occur at any intraocular pressure (IOP), elevated IOP is a modifiable risk factor, and IOP reduction remains the only clinically validated therapy [3]. Because late glaucomatous changes-optic nerve head cupping and visual field deficits-are secondary to retinal ganglion cell (RGC) atrophy and likely correlate with vision loss [4], early detection and novel approaches to glaucoma diagnosis and management remain atop the challenges facing clinicians today.

Steady-state pattern electroretinography (sspERG) is a tool that enables assessment of RGC function from the central $10-15^{\circ}$ of the fovea, providing in vivo, electrophysiological measurements that are objective, rapid, repeatable, and non-invasive. Prior studies evaluating sspERG show functional abnormalities in ocular hypertension (OHT), glaucoma suspect, and open angle glaucoma (OAG) patients prior to clinically evident structural RGC changes [5-15]. Clinical standards for ss-pERG yield reliable information [16-18] and are beneficial as an adjunct in stratifying risk/managing glaucoma suspects [11, 19]. Additionally, intraocular pressure (IOP)-lowering medications $[19,20]$ and surgery $[21,22]$ in OAG patients 
with uncontrolled IOP have shown RGC function improvements in response to treatment, as measured by ss-pERG.

The ss-pERG's role in early glaucomatous states is combined in this study with a novel device known as the multi-pressure dial or MPD (Equinox Ophthalmic, Inc., New Port Beach, CA, USA). The MPD consists of a pair of goggles that separately encloses the periorbital areas of each eye that are individually connected to a programmable pump (Fig. 1). After a target negative pressure is programmed into the device, a negative pressure microenvironment is created, which results in a corresponding decrease in IOP. The principle of operation of the device is based on Pascal's law: pressured applied to an enclosed fluid is transmitted equally through the fluid. Since the eye is essentially filled with a non-compressible fluid, pressures applied to or removed from the surface of the eye will be equally distributed throughout the eye. The MPD's mechanism for IOP reduction has been described in detail in a previous study by Ethier et al. [23]. Clinical studies have demonstrated the safety and tolerability of the MPD for up to $8 \mathrm{~h}$ of wear $[24,25]$.

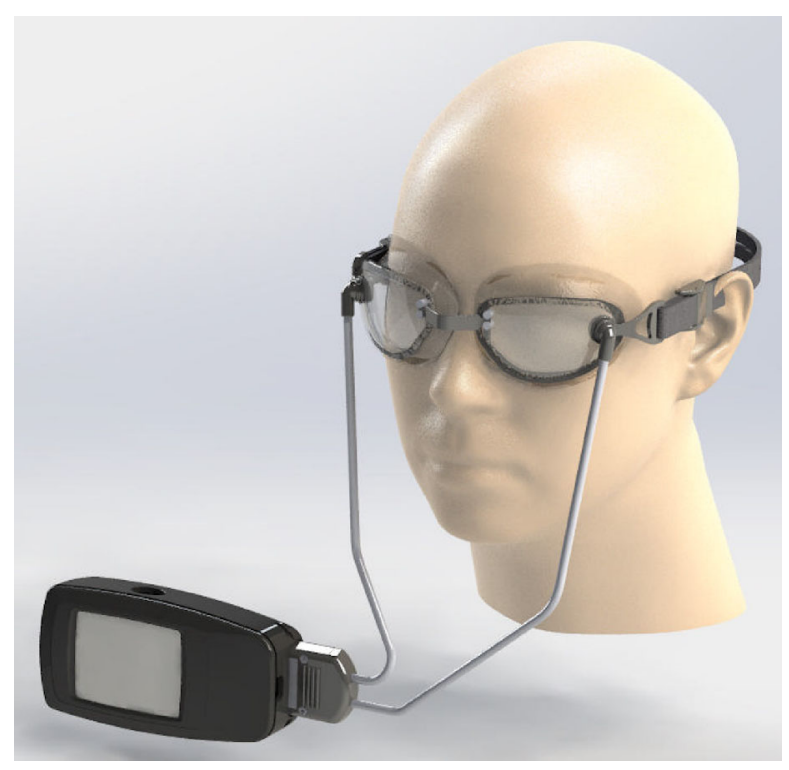

Fig. 1 Standard iteration of the multi-pressure dial (MPD), which consists of a pair of pressure-sensing goggles connected to a pressure-modulating pump
The goal of this study was to evaluate sspERG, via MagnitudeD (MagD) and MagnitudeD/Magnitude (MagD/Mag ratio), while subjects wore the MPD with negative pressure application for $2 \mathrm{~h}$, to determine if there were changes in ss-pERG parameters when IOP was lowered. We hypothesized that the MPD was capable of acutely improving the MagD and MagD/Mag ratio on ss-pERG in patients with OHT, glaucoma suspects, and mild OAG.

\section{METHODS}

\section{Design}

This was a randomized, controlled, prospective, single-site, pilot study in a private practice setting. The study included nine subjects with OHT, glaucoma suspect, or mild OAG, as defined by the American Academy of Ophthalmology Preferred Practice Pattern criteria. All procedures conducted were in accordance with the Aspire IRB and the 1964 Helsinki Declaration and its later amendments or comparable ethical standards. Informed consent was obtained for each subject prior to the study. This study was approved and designated as a non-significant risk clinical study by an institutional review board (IRB) and did not require clinical trial registration.

\section{Subjects}

Key inclusion criteria were subjects at least 18 years of age with orbital anatomy allowing proper MPD seal and the same documented diagnoses in both eyes of either OHT, glaucoma suspect, or mild OAG with Snellen BCVA of $20 / 40$ or better at 20 feet. Subjects with a history of prior eye surgery, including refractive surgery, cataract surgery, or any prior filtering surgery were included in the study. There were no subjects included in the study with a history of prior filtering surgery. Three subjects had a history of bilateral refractive surgery. Two subjects had bilateral cataract surgery, one of which also had one trabecular microbypass stent implanted in each eye. 
Exclusion criteria included the presence/history of any ocular/medical disease/condition that could interfere with the assessment of the study results or subject safety. Subjects with a history of demyelinating or seizure disorder, untreated retinal detachment/tear, untreated macular degeneration, conjunctival chemosis/ eyelid edema/eye infection in either eye were excluded from the study. Women who were pregnant or lactating during the time of the study were also excluded. Subjects with a prior ss-pERG on record were recruited from a private practice in Dakota Dunes, SD, USA. In total, twenty cases were screened: eight excluded with moderate glaucoma and three declined participation. Signed written informed consent was obtained from all nine eligible subjects prior to study enrollment.

\section{Multi-Pressure Dial}

The multi-pressure dial or MPD (Equinox Ophthalmic, Inc., New Port Beach, CA, USA) consists of two distinct components: pressuresensing goggles and a pressure-modulating pump (Fig. 1). When the goggles are worn over the eyes of a subject, negative pressure (or vacuum) is applied to create a localized decrease in atmospheric pressure contacting the surface of the eye. After a target negative pressure is set and the goggles are properly fitted to achieve a sealed environment, the negative pressure microenvironment leads to a corresponding reduction in IOP. The target negative pressure setting in the pump can be programmed separately for each periorbital region. Personnel responsible for fitting and other technical aspects of the MPD received additional training with the device and equipment. Technicians entered in desired negative periocular pressure values, specific to each subject's baseline Goldmann IOP, with the target negative pressure setting $50 \%$ of the subject's baseline IOP (e.g., negative periocular pressure target set to $-10 \mathrm{mmHg}$ for baseline IOP of $20 \mathrm{mmHg}$ ). Early clinical studies and mathematical models have demonstrated that approximately $50-60 \%$ of the negative pressure dialed into the goggles is translated as IOP reduction to the eye [23].

\section{Steady-State Pattern Electroretinography (ss-pERG)}

The Diopsys ss-pERG (Diopsys, Pine Brook, NJ, USA) High Contrast Sensitivity protocol and associated standard operating conditions were used. One trained operator performed all sspERG tests following ss-pERG training from Diopsys. To specifically elicit RGC functioning, 64 bars in an alternating black and white highcontrast $(85 \%$, corresponding to a mean luminance of $112.3 \mathrm{~lx}$ ) pattern were flashed at a reversal rate of 7.5 times per second with a frequency of $15 \mathrm{~Hz}$. Stimulation was monocular after occlusion of the other eye. Testing with excess artifacts ( $\geq 6$ per test) and inadequate signal strength (per technician discretion) were removed and repeated. The ss-pERG results are given in a waveform, which was analyzed using the Diopsys software and provided four variables of interest that were subsequently analyzed: number of artifacts, signal-to-noise ratio (SNR), MagD, and MagD/Mag ratio.

MagD/Mag ratio and MagD values are used clinically to monitor RGC function overtime. MagD/Mag ratio is defined mathematically as MagD divided by Mag, where MagD (in milliseconds) is the timing of the RGC response, and Mag (in microvolts) represents the strength of the RGC response to the checkerboard light stimulus. Taken within the clinical context, without MPD on, the manufacturer's general instructions for the High Contrast Sensitivity protocol are that absolute values of $\mathrm{MagD} / \mathrm{Mag}$ ratios $>0.752$ (and $\mathrm{MagD}>0.752$ ) indicate healthy RGC function while absolute values of MagD/Mag ratios < 0.642 (and MagD < 0.450) demonstrate concern for RGC functionality loss. Because the ss-pERG tests carried out for this study were taken while the subject continued to wear the MPD, the difference (rather than the absolute value) of MagD and MagD/ Mag ratio from each protocol stage-to-stage was considered.

\section{Protocol}

The seven-stage protocol was followed for every subject (Table 1 ). All subjects were individually 
Table 1 This table demonstrates the visit schedule for each subject and the procedures included for each stage

\begin{tabular}{|c|c|c|c|c|c|c|c|}
\hline & Stage 0 & Stage 1 & Stage 2 & Stage 3 & Stage 4 & Stage 5 & Stage 6 \\
\hline $\begin{array}{l}\text { Stage } \\
\text { description }\end{array}$ & $\begin{array}{l}\text { Baseline } \\
\text { ss-ERG } \\
\text { without } \\
\text { MPD }\end{array}$ & $\begin{array}{l}\text { Baseline ss- } \\
\text { ERG with } \\
\text { MPD } \\
\text { (negative } \\
\text { pressure } \\
\text { off) }\end{array}$ & $\begin{array}{l}\text { ss-ERG } \\
\text { with } \\
\text { MPD } \\
\text { (negative } \\
\text { pressure } \\
\text { on) }\end{array}$ & $\begin{array}{l}\text { ss-ERG after } \\
15 \text { min with } \\
\text { MPD } \\
\text { (negative } \\
\text { pressure on) }\end{array}$ & $\begin{array}{l}\text { ss-ERG after } \\
2 \mathrm{~h} \text { with } \\
\mathrm{MPD} \\
\text { (negative } \\
\text { pressure } \\
\text { on) }\end{array}$ & $\begin{array}{l}\text { ss-ERG } \\
\text { following } 2 \mathrm{~h} \\
\text { with MPD } \\
\text { (negative } \\
\text { pressure off) }\end{array}$ & $\begin{array}{l}\text { Final } \\
\text { without } \\
\text { MPD }\end{array}$ \\
\hline MPD wear & & $\mathrm{X}$ & $X$ & $X$ & $\mathrm{X}$ & $\mathrm{X}$ & \\
\hline $\begin{array}{l}\text { Negative } \\
\text { pressure } \\
\text { application } \\
\text { (vacuum) } \\
\text { active }\end{array}$ & & & $\mathrm{X}$ & $\mathrm{X}$ & $\mathrm{X}$ & & \\
\hline $\begin{array}{l}\text { Informed } \\
\text { consent }\end{array}$ & $\mathrm{X}$ & & & & & & \\
\hline $\begin{array}{l}\text { MPD seal } \\
\text { check }\end{array}$ & $\mathrm{X}$ & $\mathrm{X}$ & $\mathrm{X}$ & $\mathrm{X}$ & $\mathrm{X}$ & $\mathrm{X}$ & \\
\hline $\mathrm{BCVA}$ at $2^{\prime}$ & $\mathrm{X}$ & & & & & & \\
\hline HVF 24-2 & $\mathrm{X}$ & & & & & & \\
\hline OCT/RNFL & $\mathrm{X}$ & & & & & & \\
\hline IOP check & $\mathrm{X}$ & & & & & & \\
\hline $\begin{array}{l}\text { Slit-lamp } \\
\text { examination }\end{array}$ & $\mathrm{X}$ & & & & & & $\mathrm{X}$ \\
\hline ss-pERG & $\mathrm{X}$ & $\mathrm{X}$ & $\mathrm{X}$ & $\mathrm{X}$ & $\mathrm{X}$ & $\mathrm{X}$ & $\mathrm{X}$ \\
\hline
\end{tabular}

Stages 2-4 indicate MPD wear with vacuum on, and stages 1 and 5 indicate MPD wear with no vacuum applied $M P D$ multi-pressure dial, $B C V A$ best corrected visual acuity, HVF Humphrey visual field, $O C T / R N F L$ optical coherence tomography/retinal nerve fiber layer, $I O P$ intraocular pressure, $s s-p E R G$ steady-state pattern electroretinogram

fitted to ensure the MPD was capable of achieving a vacuum-tight seal. The MPD was then removed, and all subjects underwent a basic, undilated slit-lamp examination that included the components listed in stage 0 . To conclude stage 0 , the subject underwent a baseline ss-pERG testing. The MPD was then securely placed on the subject, with one eye of each subject randomized to receive application of negative periocular pressure, while the contralateral eye served as the intrasubject control through the goggle without negative pressure.
Negative periocular pressure was programmed to target $50 \%$ of baseline Goldmann IOP for each study eye. Each stage gathered ss-pERG information from both eyes.

The stage 1 ss-pERG was obtained immediately after secure goggle placement without vacuum application. The stage 2 ss-pERG was taken immediately after the negative periocular pressure was applied to the study eye, and the target negative periocular pressure value was logged. The stage 3 ss-pERG was obtained 15 $( \pm 10) \mathrm{min}$ following negative periocular 
pressure application to the study eye. The stage 4 ss-pERG was taken $2( \pm 1) \mathrm{h}$ following negative periocular pressure application to the study eye. Following the stage 4 ss-pERG, the vacuum application in the study eye was turned off, and the MPD remained securely placed. After 0-10 min with the MPD still placed and no negative periocular pressure application, the stage 5 ss-pERG test was conducted. With the MPD removed, the final ss-pERG was obtained during stage 6.

During application of negative periocular pressure throughout stages 2,3 , and 4 , the sspERG procedure was repeated for stages 2,3 , and 4 . When the difference between the sspERG's two MagD/Mag ratios was $\geq 0.1$, the sspERG was repeated a third time; the median MagD/Mag ratio from these three ss-pERG measurements was used for data analysis. However, when the difference between the initial two ss-pERG's MagD/Mag ratios was $<0.1$, the ss-pERG was not repeated a third time; the average between the two MagD/Mag ratios was used for data analysis.

Furthermore, in between stages 3 and 4, in an effort to offset patient fatigue, patients were offered the opportunity to walk around the office with the MPD securely on for up to $30 \mathrm{~min}$, supervised by a trained research staff member. Furthermore, if vacuum seal was lost (indicated by MPD sounding a tone) at any time during a time when vacuum should be on, subjects were instructed to adjust the MPD to reestablish the seal (indicated by absence of MPD tone). Research staff were present to assist subjects in re-achieving a proper MPD seal.

\section{Statistical Analysis}

A Wilcoxon signed rank sum test procedure was conducted to compare the population distributions between control and study groups at different time points. The significance level was set at 5\%. All data analyses were carried out using SAS 9.4 software (Cary, NC, USA).

\section{RESULTS}

The baseline characteristics of the nine subjects included in this study are summarized in Table 2. At baseline, prior to wear of the device, the mean study eye IOP was $17.4 \mathrm{mmHg}$ and $17.3 \mathrm{mmHg}$ in the control eye. The mean negative periocular pressure applied to the study eye was $-8.8 \mathrm{mmHg}$, with a range of -5 to $-12 \mathrm{mmHg}$.

The mean number of ss-pERG artifacts was 0.85 in study eyes and 0.75 in control eyes, regardless of MPD wear status, indicating low interference and reliable results obtained. For the control eyes, the average SNR was 1.1 decibels ( $\mathrm{dB}$ ) during MPD wear and $2.6 \mathrm{~dB}$ prior to/ after MPD wear. For the study eyes, the average SNR was $1.6 \mathrm{~dB}$ during MPD wear and $2.8 \mathrm{~dB}$ prior to/after MPD wear. Thus, while there was an expected slight decrease in SNR during MPD wear, taken collectively, the number of artifacts and SNR values for each ss-pERG indicate that an acceptable amount of data was collected.

Table 3 and Fig. 2 demonstrate the change in MagD from stage 2 through stage 5, as measured from the stage $1 \mathrm{MagD}$ baseline zero value. The mean MagD zero values were 0.60 for the control eyes and 0.51 for the study eyes, and the differences in these stage 1 values were not statistically significant $(p=0.13)$. From these starting MagD values, net change from the zero value was recorded. From stages 1 to 2 , the MagD value was noted to improve $(+0.10)$ in the study eyes following vacuum application. No change was seen in the control eyes during this initial application of negative periocular pressure, and the difference compared to the study eyes was not significant $(p=1.0)$. During stage 3, the MagD value dipped to 0.03 below stage 1 baseline MagD value in the study eyes; the control eyes dropped 0.11 below the same zero value. Stage 4 showed a statistically significant improvement in MagD from baseline stage $1(0.17$ versus $-0.26 ; p=0.023)$.

Table 4 and Fig. 3 demonstrate the change in MagD/Mag ratio from stage 2 through stage 5, as measured from the stage $1 \mathrm{MagD} / \mathrm{Mag}$ ratio baseline zero value. The mean MagD/Mag ratio zero values were 0.57 for the control eyes and 
Table 2 Subject demographics

\begin{tabular}{lllll}
\hline Subject & Age (years) & Diagnosis & Snellen BCVA (study eyes) & Snellen BCVA (control eyes) \\
\hline 1 & 69 & POAG, mild & $20 / 25$ & $20 / 25$ \\
2 & 73 & Glaucoma suspect & $20 / 40$ & $20 / 30$ \\
3 & 42 & POAG & $20 / 40$ & $20 / 30$ \\
4 & 57 & OAG, borderline & $20 / 30$ & $20 / 30$ \\
5 & 72 & OAG & $20 / 40$ & $20 / 30$ \\
6 & 69 & Glaucoma suspect & $20 / 30$ & $20 / 20$ \\
7 & 66 & OAG, borderline & $20 / 20$ & $20 / 20$ \\
8 & 63 & POAG & $20 / 20$ & $20 / 25$ \\
9 & 66 & POAG, mild & $20 / 20$ & $20 / 20$ \\
\hline
\end{tabular}

$B C V A$ best corrected visual acuity without trial frames or contact lenses, $P O A G$ primary open-angle glaucoma

Table 3 MagD non-parametric statistics summary during MPD wear (stages 1-5)

\begin{tabular}{lllll}
\hline Stage & MagD control eyes $(\boldsymbol{n}$, mean $\pm \mathbf{S E})$ & MagD study eyes $(\boldsymbol{n}$, mean $\pm \mathbf{S E})$ & $\boldsymbol{p}$ value & Significance \\
\hline 1 & $9,0.60 \pm 0.10$ & $9,0.51 \pm 0.11$ & 0.129 & Non-significant \\
2 & $9,0.60 \pm 0.10$ & $9,0.61 \pm 0.10$ & 1.0 & Non-significant \\
3 & $8,0.49 \pm 0.09$ & $8,0.48 \pm 0.12$ & 0.867 & Non-significant \\
4 & $9,0.34 \pm 0.03$ & $9,0.68 \pm 0.17$ & $0.023^{* *}$ & Significant \\
5 & $8,0.43 \pm 0.09$ & $8,0.49 \pm 0.12$ & 0.945 & Non-significant \\
\hline
\end{tabular}

The $p$ values are based on Wilcoxon signed rank sum test procedure. There was a statistically significant $\left(p=0.023^{* *}\right)$ difference observed between study and control eye groups at stage 4

0.44 for the study eyes, and the differences in these stage 1 values were not statistically significant $(p=0.078)$. From these starting MagD/ Mag ratios, net change from the zero value was recorded. From stages 1 to 2 , instantaneous improvement in the study eyes' MagD/Mag ratio was noted following vacuum application. No change was seen in the control eyes during this initial application of negative periocular pressure, and the difference compared to the study eyes was not significant $(p=1.0)$. The MagD/Mag ratio decreased by 0.08 in the study eye from stage 2 to stage 3 but still maintained a positive change from stage 1 zero value. Stage 4 showed improvements approaching significance in MagD/Mag ratio from baseline (0.14 versus $-0.16 ; p=0.059)$. After the negative periocular pressure application was turned off, the MagD/Mag ratio decreased by 0.10 in the study eyes but again maintained a positive change from the stage $1 \mathrm{MagD} / \mathrm{Mag}$ ratio baseline zero value.

The ss-pERG measurements during MPD wear caused the absolute value of the MagD and MagD/Mag ratios to decrease in amplitude as demonstrated from stage 0 to stage 1 and from stage 5 to stage 6 . In stages $0,1,5$, and 6 , the control eye MagD values were $0.74,0.60,0.43$, and 1.1 (and corresponding MagD/Mag ratio values were $0.66,0.57,0.46$, and 0.73 ), respectively. In stages $0,1,5$, and 6 , the study eye MagD values were $0.85,0.51,0.49$, and 1.1 (and 


\section{Mean $\triangle$ MagD Relative to Baseline}

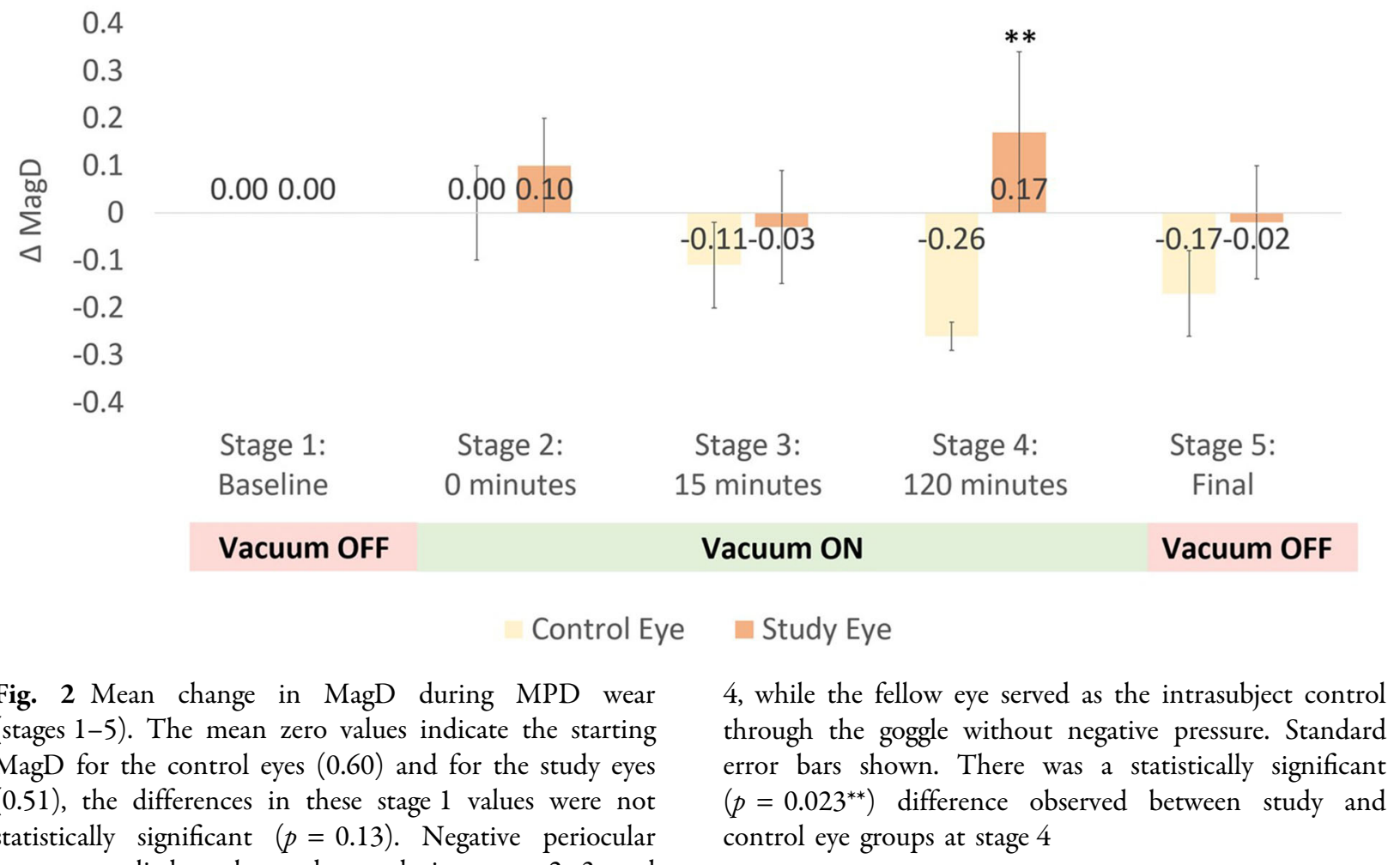

Table $4 \mathrm{MagD} / \mathrm{Mag}$ ratio non-parametric statistics summary during MPD wear (stages 1-5)

\begin{tabular}{lllll}
\hline Stage & $\begin{array}{l}\text { MagD/Mag ratio control eyes }(\boldsymbol{n}, \\
\text { mean } \pm \text { SE) }\end{array}$ & $\begin{array}{l}\text { MagD/Mag ratio study eyes }(\boldsymbol{n}, \\
\text { mean } \pm \text { SE })\end{array}$ & $\boldsymbol{p}$ value & Significance \\
\hline 1 & $9,0.57 \pm 0.04$ & $9,0.44 \pm 0.07$ & 0.078 & Non-significant \\
2 & $9,0.57 \pm 0.05$ & $9,0.57 \pm 0.07$ & 1.0 & Non-significant \\
3 & $8,0.52 \pm 0.06$ & $8,0.49 \pm 0.11$ & 0.843 & Non-significant \\
4 & $9,0.41 \pm 0.03$ & $9,0.58 \pm 0.07$ & $0.059^{*}$ & $\begin{array}{c}\text { Approached } \\
\text { significant }\end{array}$ \\
& & & & Son-significant \\
\hline
\end{tabular}

The $p$ values are based on Wilcoxon signed rank sum test procedure. The difference observed between study and control eye groups at stage 4 approached significance $(p=0.059)^{*}$

corresponding MagD/Mag ratio values were DISCUSSION $0.67,0.44,0.49$, and 0.57$)$, respectively.

This study is the first of its kind evaluating the short-term ss-pERG changes of an MPD's periocular negative pressure application in 


\section{Mean $\Delta$ MagD/Mag Ratio Relative to Baseline}

\begin{abstract}
0.3

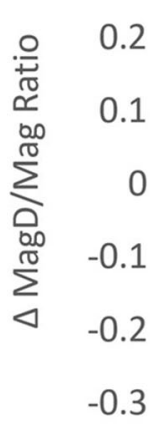

Stage 1:

Baseline

Stage 2:

0 minutes

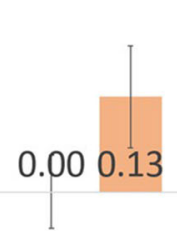

Vacuum OFF

Fig. 3 Mean Change in MagD/Mag ratios during MPD wear (stages 1-5). The mean zero values indicate the starting MagD/Mag ratio for the control eyes (0.57) and for the study eyes (0.44), and the differences in these stage 1 values were not statistically significant $(p=0.078)$. Negative periocular pressure applied to the study eye
\end{abstract}

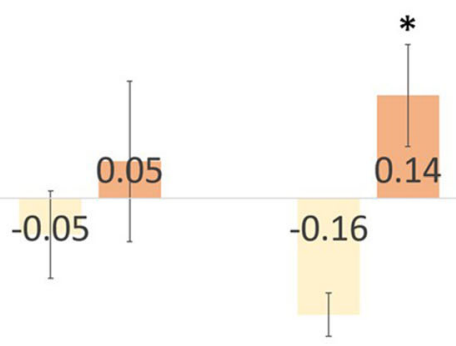

Stage 3:

15 minutes

\section{Stage 4: 120 minutes}

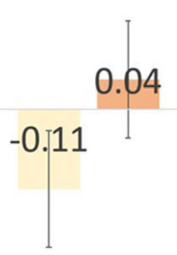

Stage 5:

Final

\section{Vacuum ON}

Vacuum OFF

\section{Control Eye $\square$ Study Eye}

glaucomatous eyes. The results of this study indicate that short-term wear of the MPD, and subsequent IOP reduction, can produce an acute, statistically significant $(p=0.023)$ improvement in the MagD value evident on sspERG, suggesting an acute improvement in RGC function. Recent studies have demonstrated the MagD value to be the most accurate and valuable ss-pERG parameter for discerning glaucomatous dysfunction [12]. There was also an improvement in the MagD/Mag ratio parameter, but the difference did not reach statistical significance $(p=0.059)$, likely because of small sample size. These findings indicate that ss-pERG may have utility in screening for early stages of glaucomatous disease, and MPD may emerge as a novel approach to treating the functional changes observed in glaucomatous eyes before progression to RGC structural changes. The acute change in ss-pERG parameters following IOP reduction observed in this study is akin to what occurs in during stages 2, 3, and 4, while the fellow eye served as the intrasubject control through the goggle without negative pressure. Standard error bars shown. The difference observed between study and control eye groups at stage 4 approached significance $(p=0.059)^{*}$

glaucomatous eyes in response to IOP-lowering drops [5, 19] and surgery [21, 22].

During ss-pERG, subjects foveate on a high contrast stimulus to maximally activate the majority of RGC, which are primarily located in the macula. Injury to selectively vulnerable RGC in glaucomatous optic neuropathies [26] is thought to occur at the lamina cribrosa (LC), a collagenous membrane with elastic properties separating intraocular vitreous anteriorly from cerebrospinal fluid (CSF) in the optic nerve sheath subarachnoid space posteriorly $[27,28]$. Because the LC is impermeable to vitreous and CSF, RGC axons passing through the LC are subject to the forces across it, namely IOP and optic nerve sheath subarachnoid space pressure (or intracranial pressure). This pressure differential across the LC is known as the translaminar pressure gradient (TLPG) and has been implicated in the pathogenesis of glaucoma [29-33]. Thus, capturing information about 
RGC function, as is done with ss-pERG, is a tool for clinicians monitoring glaucomatous change.

When other diagnostic tests, such as OCT and HVF, are normal or inconclusive, attenuation of the ss-pERG signal is a tool for identifying early glaucomatous changes in RGC function [11]. Early in the disease process, sspERG has been shown to be reversible with treatments aimed at lowering IOP. In early stages of glaucomatous diseases, reversible parameters noted in the cited literature include pERG amplitude and phase. The pERG amplitude and phase parameters are incorporated by the Diopsys ERG technology when formulating the clinical values for Mag, MagD, and MagD/ Mag ratio [5, 14, 15, 19]. Prior reports support ss-pERG as a "glaucoma detector" before permanent structural or functional changes are observed on other diagnostic modalities. However, further investigation is needed to compare the improved ss-pERG MagD and MagD/Mag ratios observed after $2 \mathrm{~h}$ of MPD IOP reduction treatment to those of current glaucoma treatments: medical therapy, laser treatments, minimally invasive glaucoma surgery (MIGS), and traditional glaucoma surgeries.

This pilot study is not without limitations, including a small sample size and ss-pERG interference from the MPD lens. Augmenting statistical power in future investigations with data from more eyes will likely help to further elucidate the extent and consistency of improved ss-pERG parameters following MPD IOP reduction treatment. During MPD wear, both study eyes and control eyes demonstrated a similar decrease in the absolute value of the MagD and MagD/Mag ratios, but it was assumed that reliable ss-pERG data was collected as supported by acceptable artifact and SNR values. Capturing the change from baseline MagD and MagD/Mag ratios values helped to account for the interference from the lens of the MPD. To build upon the preliminary results from this pilot study, primary considerations for future studies include adjusting parameters of the MPD (including increasing duration of vacuum application to study eyes) and increasing the duration between MPD IOP reduction treatment and follow-up re-evaluation. Further defining the short-term ss-pERG parameter improvements in these ways may help to provide additional insight into the MPD's lasting effects on RGC function, far beyond its IOP reduction treatment window.

Despite a small sample size, the acute change in MagD and MagD/Mag ratios suggests that an acute improvement in RGC function occurs with negative periocular pressure application. Thus, the MPD represents a potential novel treatment for glaucoma, and the positive findings of this study promote further research regarding the use of the MPD and ss-pERG, diagnostically and therapeutically.

\section{CONCLUSIONS}

Following $2 \mathrm{~h}$ of MPD wear, the measured MagD and MagD/Mag ratio improved compared to control, suggesting that negative periocular pressure application to the anterior globe can lead to a short-term improvement in one measure of retinal ganglion cell function.

\section{ACKNOWLEDGEMENTS}

We thank the study participants for their participation in the study and the American Glaucoma Society for grant support.

Funding. This study was sponsored by Equinox Ophthalmic, Inc. (New Port Beach, CA, USA), with assistance from an American Glaucoma Society grant (Mentoring for the Advancement of Physician Scientists). The Rapid Service Fees were funded by Equinox Ophthalmic, Inc. All authors had complete access to the study data and assume full responsibility for the integrity of the data and the accuracy of the data analysis.

Authorship. All named authors meet the International Committee of Medical Journal Editors (ICMJE) criteria for authorship for this article, take responsibility for the integrity of the work as a whole, and have given their approval for this version to be published. 
Disclosures. Michael Greenwood, Tanner J. Ferguson, Russell J. Swan and Brandon J. Baartman are consultants for Equinox Ophthalmic, Inc. John P. Berdahl is the founder and CEO of Equinox Ophthalmic, Inc. and holds patents licensed to Equinox Ophthalmic, Inc. Jeremy J. Kudrna, Steven Ferguson, Adam R. Bleeker and Ramu G. Sudhagoni report no conflicts of interest.

Compliance with Ethics Guidelines. All procedures conducted were in accordance with the Aspire IRB and the 1964 Helsinki Declaration and its later amendments or comparable ethical standards. Informed consent was obtained for each subject prior to the study. This study was approved and designated as a non-significant risk clinical study by an IRB and did not require clinical trial registration.

Data Availability. The datasets generated during and/or analyzed during the current study are available from the corresponding author on reasonable request.

Open Access. This article is licensed under a Creative Commons Attribution-NonCommercial 4.0 International License, which permits any non-commercial use, sharing, adaptation, distribution and reproduction in any medium or format, as long as you give appropriate credit to the original author(s) and the source, provide a link to the Creative Commons licence, and indicate if changes were made. The images or other third party material in this article are included in the article's Creative Commons licence, unless indicated otherwise in a credit line to the material. If material is not included in the article's Creative Commons licence and your intended use is not permitted by statutory regulation or exceeds the permitted use, you will need to obtain permission directly from the copyright holder. To view a copy of this licence, visit http://creativecommons.org/licenses/by$\mathrm{nc} / 4.0 /$.

\section{REFERENCES}

1. Quigley HA. The number of people with glaucoma worldwide in 2010 and 2020. Br J Ophthalmol. 2006;90(3):262-7.

2. Tham Y-C, Li X, Wong TY, Quigley HA, Aung T, Cheng C-Y. Global prevalence of glaucoma and projections of glaucoma burden through 2040. Ophthalmology. 2014;121(11):2081-90.

3. Heijl A, Leske MC, Bengtsson B, Hyman L, Bengtsson B, Hussein M. Reduction of intraocular pressure and glaucoma progression: results from the early manifest glaucoma trial. Arch Ophthalmol. 2002;120(10):1268-79.

4. Cohen LP, Pasquale LR. Clinical characteristics and current treatment of glaucoma. Cold Spring Harb Perspect Med. 2014;4(6):a017236-a017236017236.

5. Ventura L, Porciatti V. Restoration of retinal ganglion cell function in early glaucoma after intraocular pressure reduction: a pilot study. Ophthalmology. 2005;112(1):20-7.

6. Banitt MR, Ventura LM, Feuer WJ, et al. Progressive loss of retinal ganglion cell function precedes structural loss by several years in glaucoma suspects. Investig Opthalmology Vis Sci. 2013;54(3): 2346.

7. Ventura LM, Porciatti V. Pattern electroretinogram in glaucoma. Curr Opin Ophthalmol. 2006;17(2): 196-202.

8. Bach M, Unsoeld AS, Philippin H, et al. Pattern ERG as an early glaucoma indicator in ocular hypertension: a long-term, prospective study. Invest Ophthalmol Vis Sci. 2006;47(11):4881-7.

9. Wilsey LJ, Fortune B. Electroretinography in glaucoma diagnosis. Curr Opin Ophthalmol. 2016;27(2):118-24.

10. Bach M. Electrophysiological approaches for early detection of glaucoma. Eur J Ophthalmol. 2001;11(Suppl 2):S41-49.

11. Bach M, Hoffmann MB. Update on the pattern electroretinogram in glaucoma. Optom Vis Sci. 2008;85(6):386-95.

12. Amarasekera DC, Resende AF, Waisbourd M, et al. Steady-state pattern electroretinogram and shortduration transient visual evoked potentials in glaucomatous and healthy eyes. Clin Experiment Ophthalmol. 2018;46(1):54-61.

13. Turkey E, Elsanabary ZSE, Elshazly LHM, Osman $\mathrm{MH}$. Role of pattern electroretinogram in ocular 
hypertension and early glaucoma. J Glaucoma. 2019;28(10):871-7.

14. Papst N, Bopp M, Schnaudigel OE. The pattern evoked electroretinogram associated with elevated intraocular pressure. Graefes Arch Clin Exp Ophthalmol. 1984;222(1):34-7.

15. Colotto A, Salgarello T, Giudiceandrea A, et al. Pattern electroretinogram in treated ocular hypertension: a cross-sectional study after timolol maleate therapy. Ophthalmic Res. 1995;27(3):168-77.

16. Bach $\mathrm{M}$, Brigell MG, Hawlina $\mathrm{M}$, et al. ISCEV standard for clinical pattern electroretinography (PERG): 2012 update. Doc Ophthalmol. 2013;126(1):1-7.

17. Resende AF, Sanvicente CT, Eshraghi H, et al. Test-retest repeatability of the pattern electroretinogram and flicker electroretinogram. Doc Ophthalmol. 2019;139(3):185-95.

18. Gillmann K, Mansouri K, Rao HL, et al. A prospective evaluation of the repeatability and reliability of new steady-state pattern electroretinogram parameters. J Glaucoma. 2018;27(12):1079-85.

19. Karaśkiewicz J, Penkala K, Mularczyk M, Lubiński W. Evaluation of retinal ganglion cell function after intraocular pressure reduction measured by pattern electroretinogram in patients with primary openangle glaucoma. Doc Ophthalmol. 2017;134(2): 89-97.

20. Ventura LM, Porciatti V. Restoration of retinal ganglion cell function in early glaucoma after intraocular pressure reduction: a pilot study. Ophthalmology. 2005;112(1):20-7.

21. Sehi M, Grewal DS, Goodkin ML, Greenfield DS. Reversal of retinal ganglion cell dysfunction after surgical reduction of intraocular pressure. Ophthalmology. 2010;117(12):2329-36.

22. Spadea L, Giuffrè I, Bianco G, Balestrazzi E. PERG and P-VEP after surgical trabeculectomy in primary open-angle glaucoma. Eur J Ophthalmol. 1995;5(2): 92-5.

23. Ethier CR, Yoo P, Berdahl JP. The effects of negative periocular pressure on intraocular pressure. Exp Eye Res. 2020;191:107928.
24. Samuelson TW, Ferguson TJ, Radcliffe NM, et al. 8 hrs Safety evaluation of a multi-pressure dial in eyes with glaucoma: prospective, open-label, randomized study. Clin Ophthalmol. 2019;13:1947-53.

25. Thompson VM, Ferguson TJ, Ahmed IIK, et al. Short-term safety evaluation of a multi-pressure dial: a prospective, open-label, non-randomized study. Ophthalmol Ther. 2019;8(2):279-87.

26. Della Santina L, Ou Y. Who's lost first? Susceptibility of retinal ganglion cell types in experimental glaucoma. Exp Eye Res. 2017;158:43-50.

27. Hernandez MR, Pena JDO. The optic nerve head in glaucomatous optic neuropathy. Arch Ophthalmol. 1997;115(3):389-95.

28. Hernandez MR, Andrzejewska WM, Neufeld AH. Changes in the extracellular matrix of the human optic nerve head in primary open-angle glaucoma. Am J Ophthalmol. 1990;109(2):180-8.

29. Ren R, Jonas JB, Tian G, et al. Cerebrospinal fluid pressure in glaucoma: a prospective study. Ophthalmology. 2010;117(2):259-66.

30. Ren R, Wang N, Zhang X, Cui T, Jonas JB. Translamina cribrosa pressure difference correlated with neuroretinal rim area in glaucoma. Graefes Arch Clin Exp Ophthalmol. 2011;249(7):1057-63.

31. Berdahl JP, Allingham RR, Johnson DH. Cerebrospinal fluid pressure is decreased in primary open-angle glaucoma. Ophthalmology. 2008;115(5):763-8.

32. Berdahl JP, Fautsch MP, Stinnett SS, Allingham RR. Intracranial pressure in primary open angle glaucoma, normal tension glaucoma, and ocular hypertension: a case-control study. Invest Ophthalmol Vis Sci. 2008;49(12):5412-8.

33. Baneke AJ, Aubry J, Viswanathan AC, Plant GT. The role of intracranial pressure in glaucoma and therapeutic implications. Eye (Lond). 2020;34(1): 178-91. 